\title{
Non-autologous endodontic pulp regeneration approach in molar teeth of the rat
}

\author{
U. Schenk ${ }^{\mathrm{a}}$, H. Prenzel ${ }^{\mathrm{a}}$, G. Magnucki ${ }^{\mathrm{b}}$, C. Hoang-Vu ${ }^{\mathrm{a}}$, H.G. Schaller ${ }^{\mathrm{b}}$, A. Diederich ${ }^{\mathrm{b}}$, \\ C. Gernhardt ${ }^{\mathrm{b}}$, K. Dietze ${ }^{\mathrm{c}}$, A. Navarrete Santos ${ }^{\mathrm{f}}$, S.M. Niehues ${ }^{\mathrm{d}}$, F. Jung ${ }^{\mathrm{e}}$ and B. Hiebl ${ }^{\mathrm{f}, *}$ \\ ${ }^{a}$ Clinic and Polyclinic for General Surgery, Martin-Luther-University Halle-Wittenberg, Halle (Saale), \\ Germany \\ ${ }^{\mathrm{b}}$ Clinic and Polyclinic for Operative Dentistry and Periodontology, Martin-Luther-University Halle- \\ Wittenberg, Halle (Saale), Germany \\ ${ }^{\mathrm{c}}$ Faculty of Veterinary Medicine, Institute of Veterinary Anatomy, Freie Universität Berlin, Berlin, \\ Germany \\ ${ }^{\mathrm{d}}$ Department of Radiology, Charité -University Medicine Berlin, Berlin, Germany \\ ${ }^{\mathrm{e}}$ Department of Clinical Haemostasiology and Transfusion Medicine, University of Saarland, Homburg \\ (Saar), Germany \\ ${ }^{\mathrm{f}}$ Centerfor Medical Basic Research, Martin-Luther-University Halle-Wittenberg, Halle (Saale), Germany
}

\begin{abstract}
The dental pulp is an important soft connective tissue which is able to produce dentin over time as a reaction on external stimuli. It also maintains the biological and physiological vitality of the dentin. Due to this the pulp is essential for teeth homeostasis. However, dental caries is still one of the most prevalent health problems in dentistry and therefore, one major cause for early loss of the dental pulp vitality and subsequent tooth extractions. Meanwhile the potential for successful pulp regeneration therapy is increasing due to advances in the field of regenerative endodontics. Thus, adequate experimental animal models are required for testing and validating these new regenerative therapies. Rodents and rats in particular, are relevant models for experimental periodontal research. The breeding and housing costs of rats are relatively low facilitating studies with sufficient numbers for statistical analysis in comparison to bigger sized mammals like beagle dogs, miniature pigs or monkeys. Additionally, rat molar teeth and pulps are characterized by similar anatomical, histological, biological and physiological features to human teeth. Essential biological reactions of the pulp tissue and the interaction during the different stages of wound healing of rat molar teeth are comparable to that of other mammals. However, despite of the multiple research activities in the field of regenerative endodontics and the above mentioned advantages of the rat model only rare in vivo studies are published. Therefore, the presented study aimed to introduce the rat molar teeth as a valid model for studying dental pulp stem cell based endodontic tissue regeneration. Human dental pulp stem cells were implanted into the pulp of immunodeficient rats (RNU rats). Cell growth was supported by a collagenous membrane, which was applied on top of the cells after implantation. After closing the pulpal cavity with a light-polymerisable resin human dental pulp stem cells were able to maintain cell viability in the rat molar pulp niche for at least three weeks. This demonstrated the suitability of immunodeficient RNU rats for non-autologous dental stem cell based endodontic tissue engineering approaches.
\end{abstract}

Keywords: RNU rats, dental pulp stem cells, pulp regeneration

${ }^{*}$ Corresponding author: B. Hiebl, Center for Medical Basic Research, Martin-Luther-University Halle-Wittenberg, Halle (Saale), Germany. Tel./Fax: +49 345557 1793; E-mail: bernhard.hiebl@ medizin.uni-halle.de. 


\section{Introduction}

Dental pulp facilitates teeth to grow, mature, and respond to stress. It is a soft connective tissue which is able to produce dentin, and which maintains the biological and physiological vitality of the dentin. Thus, dental pulp is essential for teeth homeostasis. However, dental caries is still one of the most prevalent health problems in dentistry [1] and a major cause for early loss of dental pulp vitality and subsequent tooth deficiency. Meanwhile the potential for successful pulp regeneration therapy is increasing due to advances in the field of regenerative endodontics [2] and appropriate experimental animal models are required for testing and validating these new regenerative therapies. Rodents and rats in particular, are relevant models for experimental periodontal and dental research $[3,4]$. The breeding and housing costs are relatively low making it possible to carry out studies with sufficient numbers from a biometrical point of view. Additionally, the rat molar teeth and pulp tissue are characterized by similar anatomical, histological, biological and physiological features [5-8] to human molar teeth. Also, essential biological reactions of the pulp tissue and the interaction during the different stages of wound healing of rat molar teeth are comparable to that of other mammals [9]. However, despite of the multiple research activities in the field of regenerative endodontics and the obvious advantages of the rat model in this research topic only rare in vivo studies are published using the rat animal model. Thus, objective of the study was to introduce the immuno-deficient nude rat [10] molar teeth as a valid model for studying xenogeneic dental pulp stem cell based endodontic tissue regeneration approaches.

\section{Materials and methods}

\subsection{Dental pulp stem cells}

Dental pulp stem cells were isolated from wisdom teeth of healthy donors ( $n=9,16-20$ years of age). The study was approved by the Research Ethics Committee of the Medical Faculty of the Martin-LutherUniversity Halle-Wittenberg. All patients provided written informed consent after interview and inclusion assessment.

Adherent connective tissue was removed from the wisdom teeth immediately after extraction by scraping before the teeth were prepared to gain access to the pulp chamber. Harvested pulp was transferred into a petri dish, grossly minced with a scalpel and incubated for $90 \mathrm{~min}$ at $37^{\circ} \mathrm{C}$ in a humidified atmosphere with a mixture $3 \mathrm{mg} / \mathrm{ml}$ collagenase type I and $4 \mathrm{mg} / \mathrm{ml}$ dispase. Subsequently cells were separated from non-cellular components with a $70 \mu \mathrm{m}$ pore size filter and were grown in $\alpha$-MEM cell culture medium supplemented with $10 \mathrm{vol} \% \mathrm{FCS}, 100 \mathrm{nM}$ ascorbic acid, $2 \mathrm{mM} \mathrm{L}$-glutamine, and $250 \mu \mathrm{g} / \mathrm{ml}$ amphotericin $\mathrm{B}$ (Fungizone ${ }^{\mathrm{TM}}$ ). Dental pulp stem cells were purified by twofold flow cytometric cell sorting (FACS Vantage, BD Biosciences) using the mesenchymal stem cell marker STRO-1 after three cell passages.

\subsection{PCR analysis}

Prior to implantation, STRO-1 cells were also tested for expression of mesenchymal stem cell markers such as CD29, CD44, CD90, CD105, and CD146. Total RNA was isolated from $3.5 \times 10^{4} / \mathrm{ml}$ of DPSCs by employment of TRIzol reagent. $1 \mu \mathrm{g}$ of total RNA was used as a template for first strand cDNA synthesis (SuperScript ${ }^{\mathrm{TM}}$ II KIT, Life Technologies) according to instructions provided by the manufacturer. Samples were stored at $-20^{\circ} \mathrm{C}$. Amplifications of CD105, CD44, CD146, CD90, CD29, and GAPDH 
were performed with Rotor-Gene Q Realtime qPCR System (Qiagen) and SYBRGreen MasterMix according to instructions provided by the manufacturer (Qiagen). The following primer pairs were used: GAPDH sense: 5'ACCCAGAAGACTGTGGATGG-3', antisense: 3'-TTCTAGACGGCAGGTCAGGT-5', CD29 sense: AACTGCACCAGCCCATTT, antisense: AGCCAATCAGTGATCCACAA, CD44 sense: GACCTCTGCAAGGCTTTCAAT, antisense: AATCACCACGTGCCCTTCTAT, CD90 sense: TCCCGAACCAACTTCACCAG, antisense: GATGCCCTCACACTTGACCA, CD105 sense: ACTCTCCAGGCATC CAAGC, antisense: GGAAGGATGGCAGCTCTGT, CD146 sense: GTCGTCCCAGACTGTGGAGT, antisense: CCACTTCCAGCCACACTTTT. GAPDH was used as housekeeping gene.

\subsection{Animal housing and care}

All animal experiments described here were approved by the Regional Council of Halle (Germany). Adult (6 weeks old, $20 \pm 4 \mathrm{~g}$ body weight, male), specific pathogen-free (FELASA) inbred and
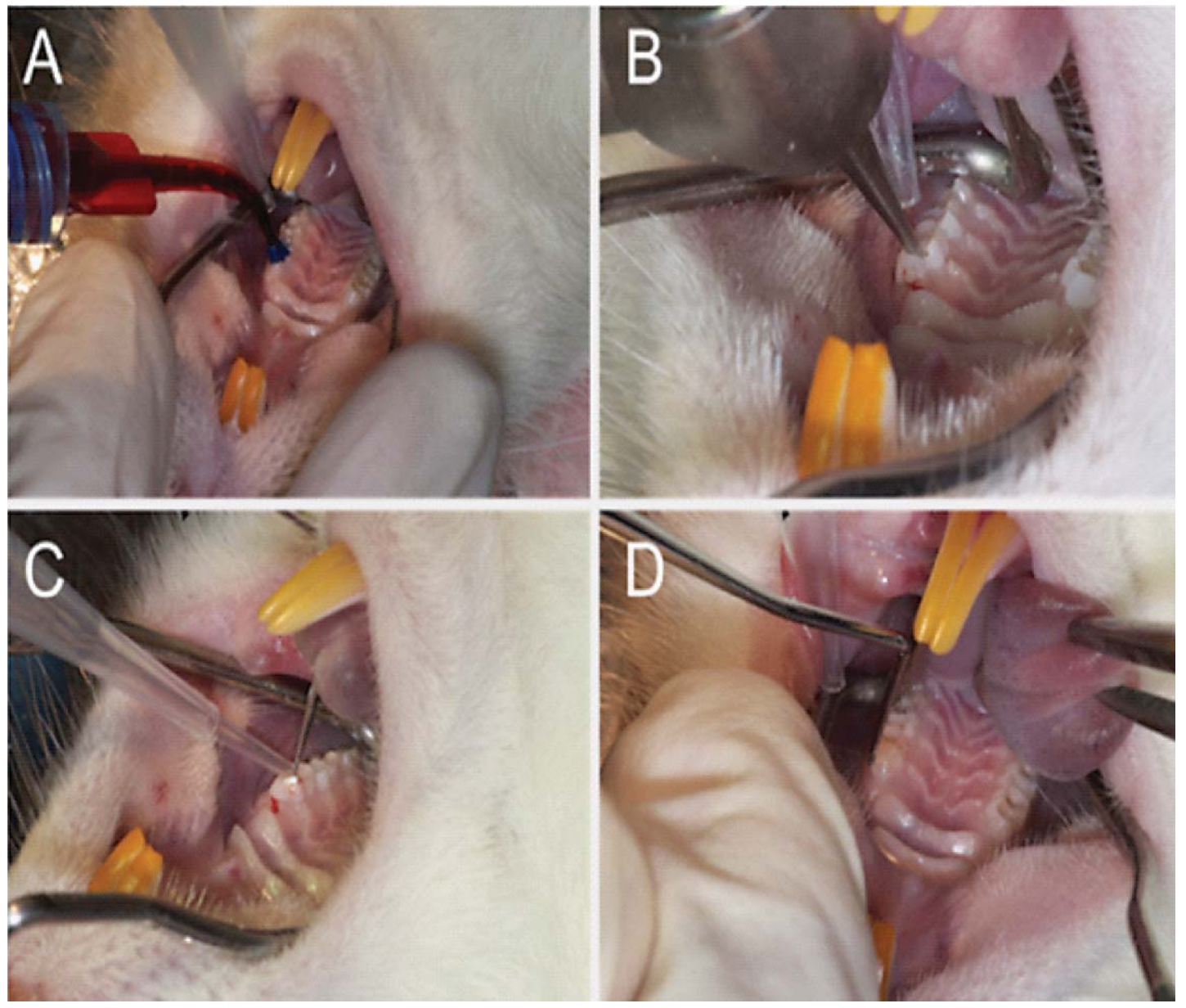

Fig. 1. Stem cell implantation into the left molar of the upper jaw of a rat: (A) etching of the occlusal surface of the first molar, (B) tooth trepanation by drilling, (C) covering the implanted cells with a collagenous membrane, (D) closing the access cavity with a light-curing dental composite resin. 
congenitally T-cell deficient rats (Rowett Nude [RNU], Crl:NIH-Foxn1 nu, $n=13$, Charles River) were subjected to xenogeneic dental stem cell transplantations. According to the European Guidelines for care and use of laboratory animals, the animals were kept at a temperature of $22 \pm 1{ }^{\circ} \mathrm{C}$ and a humidity of $65 \%$. Artificial lighting was used to maintain a regular pattern of $12 \mathrm{hrs}$ daylight and $12 \mathrm{hrs}$ night.

\subsection{Cell implantation}

For stem cell implantation, moderate surgical anesthesia was induced by intraperitoneal injection of ketamine $(75 \mathrm{mg} / \mathrm{kg})$ and medetomidine $(0.5 \mathrm{mg} / \mathrm{kg})$. Body temperature was maintained at $37^{\circ} \mathrm{C}$ during surgery by a heating blanket. The mouth was kept open by a mouth gag. Cells were implanted on top of the pulp of the first molar of the left upper jaw with the rats being in spine position. After desinfection and etching the occlusal surface of the first molar (see Fig. 1A) with 37.5 vol\% phosphoric acid, an access cavity into the pulp was drilled by a two-step trepanation procedure using drill size $0.9 \mathrm{~mm}$ in the first step and drill size $0.7 \mathrm{~mm}$ in the second step (Fig. 1B). $2 \times 10^{4}$ dental pulp stem cells suspended in $10 \mu 1$ of PBS were applied using a $21 \mathrm{G}$ injection needle. Subsequently, a porcine collagenous membrane (diameter $0.6 \mu \mathrm{m}$, Biogide, Geistlich Pharma) was placed on top of the implanted cells (Fig. 1C) and the access cavity was closed with a light-curing dental composite resin (Tetric Flow, Ivoclar Vivadent). After lightcuring of the resin material (Fig. 1D) the enamel of the crown from the antagonistic molar of the lower jaw was shortened to prevent contact with the dental filling. The same procedure was performed at the right side without insertion of cells (sham operation). Carprofen (Rimadyl ${ }^{\mathrm{TM}}$ ) was injected subcutaneously q.d. for three days $(5 \mathrm{mg} / \mathrm{kg})$ as analgesic therapy.

\subsection{Histology}

Three weeks after implantation, the animals were euthanized and upper and lower jaws were prepared for further tests. First, adherent tissue was removed grossly. Subsequently, the jaws were incubated 40 days at $4^{\circ} \mathrm{C}$ in $300 \mathrm{nM}$ EDTA for demineralization, and frozen sections were prepared afterwards. Sections were stained with to hematoxylin-eosin (HE) and Heidenhain azan according to the protocols described by Romeis [11]. Human stem cells within the rat pulp were visualized using immunostaining with anti-human nuclei IgG (Biomol, 1:100) and Alexa Fluor 594 donkey anti-mouse IgG (1:100, Life Technologies) as primary and secondary antibodies, respectively.

\subsection{X-ray imaging}

X-ray imaging was performed with a HELIODENT MD system (Siemens) using $70 \mathrm{kV}$ and an exposure time of $0.2 \mathrm{sec}$.

\subsection{Statistics}

Data were reported as mean value \pm standard deviation for continuous variables, and were analyzed by Student's $t$-test (two-sided, paired). $P$ values of less than 0.05 were considered significant. 

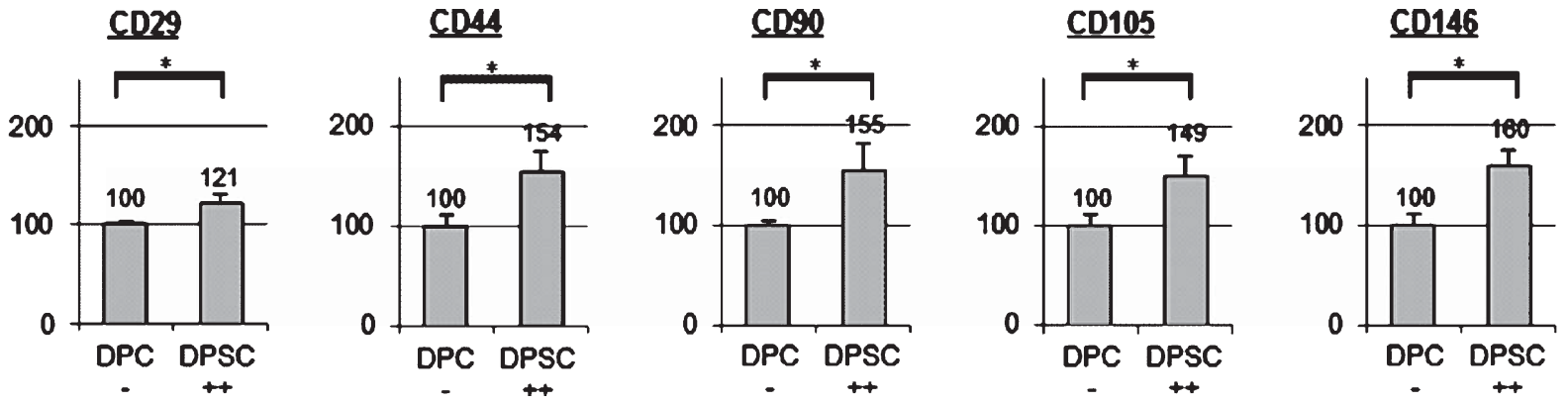

Fig. 2. Expression of markers for mesenchymal stromal cells (CD29, CD44, CD90, CD105, and CD146) in STRO-1 positive sorted pulp cells of human wisdom teeth (DPSC ++). STRO- ${ }^{-}$dental pulp cells (DPC - ) were used as control; ${ }^{*}$ indicates significant differences $(p<0.05) ;{ }^{+}+$strong expression of the $\mathrm{CD}$ markers.

\section{Results}

\subsection{Sourcing dental pulp stem cells}

Dental pulp stem cells were obtained from the pulp of human wisdom teeth by flow cytometry cell sorting using the mesenchymal stem cell marker STRO-1. Sorted STRO-1 positive cells were also tested for expression of the mesenchymal stem cell markers CD29, CD44, CD90, CD105, and CD146 prior to implantation into the rat pulp (Fig. 2).

\subsection{Implantation and growth of stem cells}

The protocol for access cavity formation in the first molar was effective for forming an adequately sized channel into the pulp for stem cell implantation (Fig. 3). $2 \times 10^{4}$ of the human cells positive for
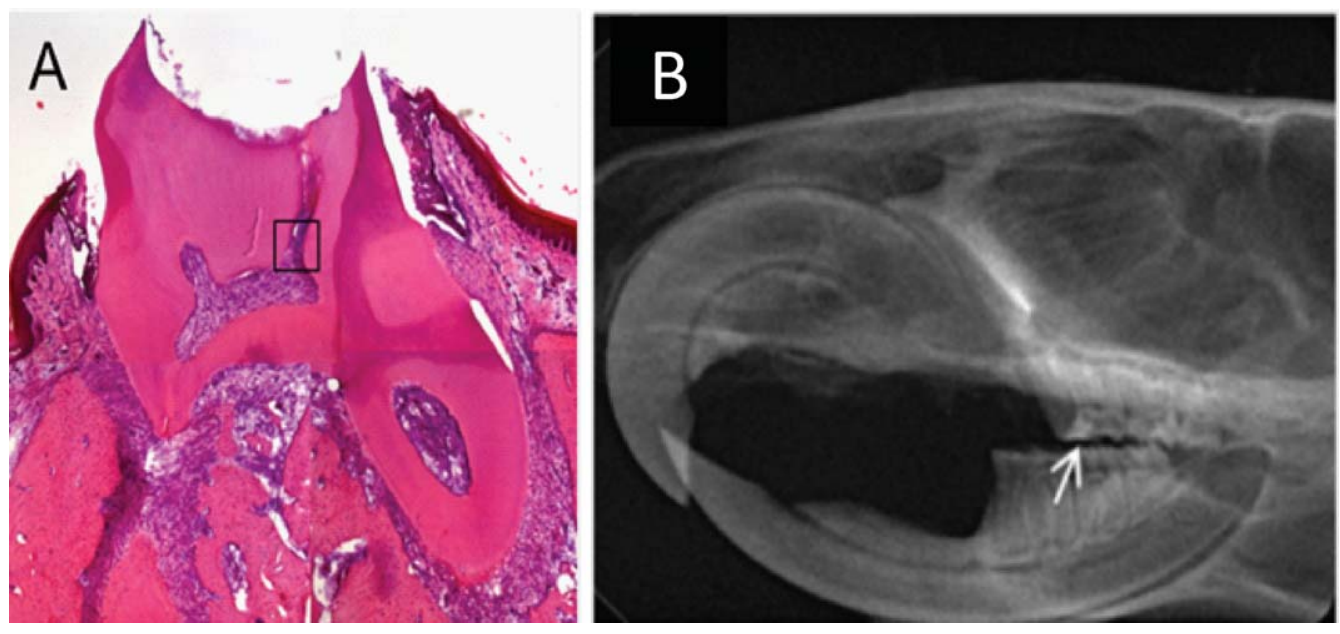

Fig. 3. First molar of an adult rat three weeks after human dental stem cell implantation into the prepared pulp cavity: (A) HE-staining of a cross section from the molar showing the access cavity into the pulp chamber with inserted resin filling (see region of interest); (B) x-ray of the lower and upper jaw, lateral view; arrow shows position of the resin filling of the first molar. 


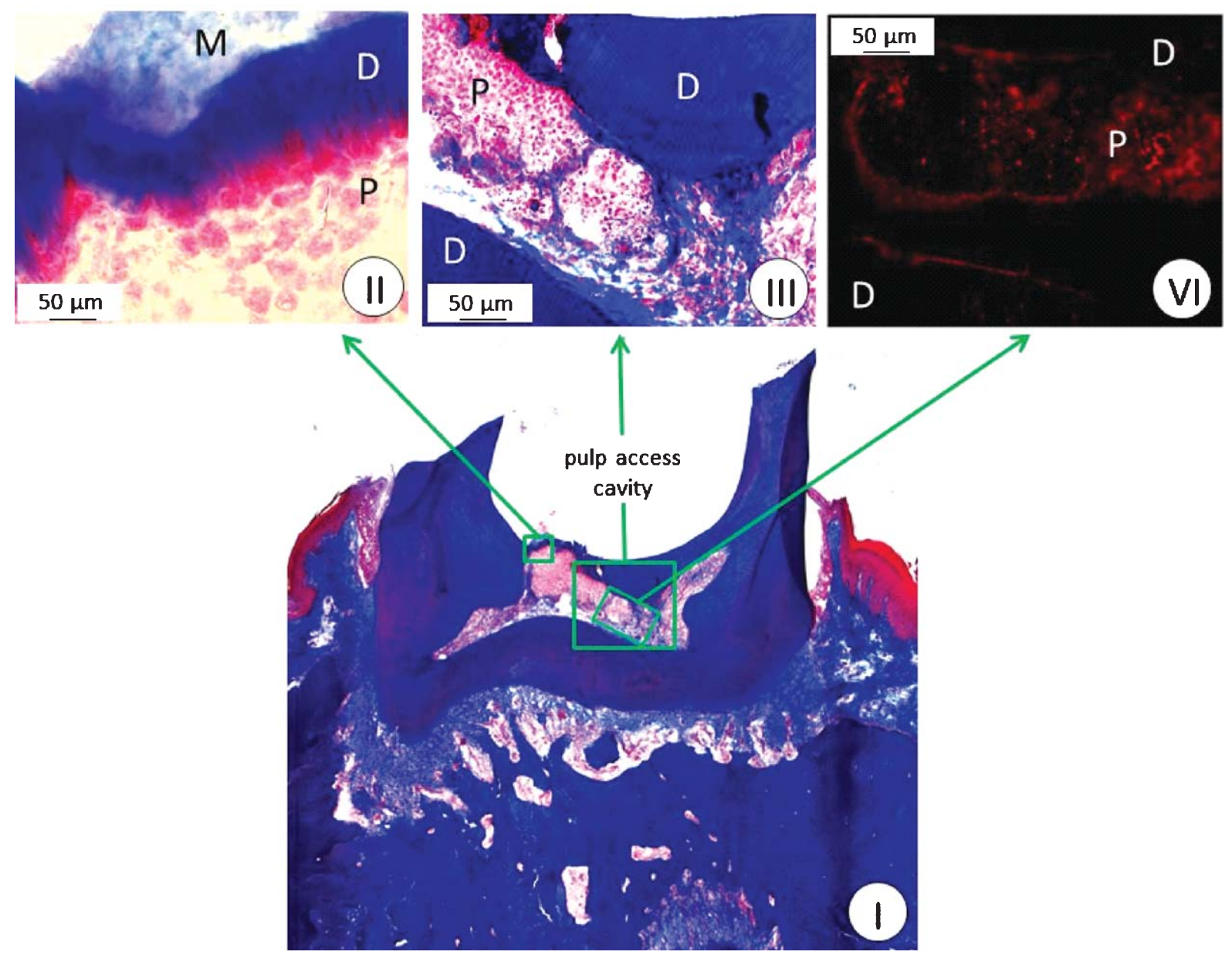

Fig. 4. (I-III) First molar in the upper jaw of a rat three weeks after human dental stem cell implantation; Heidenhain azan staining, phase contrast microscopy, M: collagenous membrane, D: dentin; P: pulp; (IV) anti-human nuclei immunostaining.

STRO-1, CD29, CD44, CD90, CD105, and CD144 were successfully implanted into the pulp (Fig. 4, I-III). Human nuclei specific immunostaining of the molar tooth cross sections showed that the implanted human cells survived in the pulp niche throughout the complete test period of three weeks (Fig. 4, VI).

Within these three weeks, the UV sensitive resin filling provided a sufficient closing of the prepared access cavity (Fig. 3B). Moreover, abrasion or cleavage formation on the occlusal surface of the molar could be observed during this period of time corrobating the quality of the process of access cavity formation and closure.

\section{Discussion}

Inflammation or necrosis of the dental pulp is one of the most common dental diseases. Currently the major therapeutical approach to maintain affected teeth is the root canal treatment with approximately 22 million root canal treatments in the United States per year [12]. During root canal therapy the inflamed 
or necrotic pulp is removed, followed by cleaning and desinfection of the root and subsequent filling of the root canal with an inert material. Although the root canal treatment offers high levels of success with rates between 78-98\% [13,14], an optimized therapeutic strategy might consist of a regenerative endodontic approach in which diseased or necrotic pulp tissue is replaced with healthy pulp tissue to revitalize teeth [15].

However, despite of approaches related to regenerative endodontics, this research field lacks of sufficient in vivo testing systems and systematic data about adequate small animal models. Thus, objective of this study was to test the practicality of the molar tooth of rats regarding this research topic. Since a protocol for isolation of human dental pulp cells had been established in 2000 [16], the study design was focused on rats which are suited for tests with xenogeneic stem cells.

At least five types of mesenchymal stem cells have been described and isolated from dental tissues, including dental pulp stem cells, stem cells of human exfoliated deciduous teeth, stem cells of the apical papilla, dental follicle progenitor cells and stem cells from periodontal ligament [17], but not all of them displayed a strong potential for pulp regeneration. However, pulp regeneration approaches using dental pulp stem cells showed promising results. The protocol used for dental stem cell isolation from human wisdom teeth was based on a stepwise flow cytometry cell sorting process with an initial sorting step using STRO-1 which is reported to be one of the best known markers for mesenchymal stem cells. The mesenchymal origin of the STRO-1 cells was also confirmed on the genomic level by expression of the marker panel CD29, CD44, CD90, CD105, and CD146.

A variety of biomaterials to support cell growth and tissue regeneration within the pulp cavity have been developed including natural polymers, synthetic polymers, hydrogels, or bioceramics. In the study presented here a collagenous membrane was applied on top of the implanted dental pulp stem cells as biomaterial, since collagen is well known to support mesenchymal cell growth and differentiation $[18,19]$. Naturally derived collagen is similar in its chemical and biological characteristics to tissues, and displays a low immunogenicity. Type I collagen which is the predominant component of the dental pulp is considered to offer initiation sites for dentin calcification [20]. Histological examinations showed maintenance of viability the human dental pulp stem cells in the rat pulp beneath the collagenous membrane for at least three weeks. After this period of time also no debris was obvious within the implant site. Therefore, we concluded that our experimental set up demonstrated the possibility to use immuno-deficient, nude rats to test regenerative endodontic approaches. However, due to the limitations of the experimental setup the study does not allow for conclusions regarding cell proliferation and cell functionality.

Further limitations are represented by the animal model itself due to differences in dentition of rats and humans. Humans are diphyodont mammals with two successive generations of teeth. Initially, there are "deciduous" teeth followed by "permanent" teeth. In contrast, the dentition of rats is monophyodont, i.e. that they have one set of teeth throughout their life. In the rat, the first molar tooth erupts on day 19 after birth, the second on day 21, and the third on day 35-40. Dentition is complete at an age of 6 weeks, and by 125 days molar growth slows significantly [21]. Moreover, the number of incisors is lower in rats than in humans, dentition of the rat is composed only of molars and incisors, and there are no canines and premolars (rat dental formula: I 1-1, C 0-0, P 0-0, M 3-3) resulting in a long toothless area between the incisors and molars. However, whereas incisors of rats are very different in structure and composition from those of humans, molars of rats are very similar to those of humans. However, a main difference represents the number of roots in the first two molars. In the upper jaw of rats the first molar has five and the second molar four roots, whereas in humans both teeth display three roots. In the lower jaw the first molar of rats is characterized by four roots and the third molar has three, whereas only two roots are present in humans [22]. Despite these differences between rat and human molars, rats might be beneficial 
for research regarding regenerative endodontics. The technology to generate transgenic rats and disease models [23] is well established for rats. Moreover, costs and effort for housing, feeding, and caring are lower in rats than in larger animals, and most antibodies required for cellular and molecular biological techniques are only available for mice and rats. However, mice are too small for most dental procedures. Even in rats these procedures require experienced personnel, since rat molars are approximately 50 times smaller than human molars [24], with molar size decreasing from the first to the third molar [22].

\section{Conclusion}

This study showed the suitability of immunodeficient RNU rats for xenogeneic dental stem cell based endodontic tissue engineering approaches. Human pulp derived dental stem cells, characterized by an established marker panel for mesenchymal stem cells including STRO-1, CD29, CD44, CD90, CD105, and CD144 maintained cell viability in the rat pulp for at least three week. Further studies are needed to show that these stem cells also proliferate and produce dentin in the rat pulp niche.

\section{References}

[1] Bagramian RA, Garcia-Godoy F, Volpe AR. The global increase in dental caries. A pending public health crisis. Am J Dent 2009;22:3-8.

[2] Demarco FF, et al. Dental pulp tissue engineering. Braz Dent J 2011;22:3-13.

[3] Dannan A, Alkattan F. Animal models in periodontal research: A mini-review of the literature. The Internet Journal of Veterinary Medicine 2007;5.

[4] Struillou X, et al. Experimental animal models in periodontology: A review. Open Dent J 2010;4:37-47.

[5] Kiryati AA. The effect of hydrocortisone plus polyantibiotics upon the damaged and infected dental pulp of rat molars. J Dent Res 1958;37:886-901.

[6] Kozlov M, Massler M. Histologic effects of various drugs on amputated pulps of rat molars. Oral Surg Oral Med Oral Pathol 1960;13:455-69.

[7] Maurice CG, Schour I. Experimental cavity preparations in the molar of the rat. J Dent Res 1955;34:429-34.

[8] Sasaki T, Kawamata-Kido H. Providing an environment for reparative dentine induction in amputated rat molar pulp by high molecular-weight hyaluronic acid. Arch Oral Biol 1995;40:209-19.

[9] Dammaschke T. Rat molar teeth as a study model for direct pulp capping research in dentistry. Lab Anim 2010;44:1-6.

[10] Hougen HP. The athymic nude rat. Immunobiological characteristics with special reference to establishment of non-antigenspecific T-cell reactivity and induction of antigen-specific immunity. APMIS 1991;(Suppl 21):1-39.

[11] Mulisch M, Welsch U, eds. Romeis Mikroskopische Technik. 18 ed. 2010, Spektrum: Heidelberg.

[12] A.D. Association, The 2006 survey of dental services rendered. American Dental Association 2007;44.

[13] Torabinejad $\mathrm{M}$, et al. Outcomes of nonsurgical retreatment and endodontic surgery: A systematic review. J Endod 2009;35:930-7.

[14] Ng YL, Mann V, Gulabivala K. Tooth survival following non-surgical root canal treatment: A systematic review of the literature. Int Endod J 2010;43:171-89.

[15] Huang GT. Dental pulp and dentin tissue engineering and regeneration: Advancement and challenge. Front Biosci (Elite Ed) 2011;3:788-800.

[16] Gronthos S, et al. Postnatal human dental pulp stem cells (DPSCs) in vitro and in vivo, Proc Natl Acad Sci U S A 2000;97:13625-30.

[17] Huang GT, Gronthos S, Shi S. Mesenchymal stem cells derived from dental tissues vs. those from other sources: Their biology and role in regenerative medicine. J Dent Res 2009;88:792-806.

[18] Zhang W, et al. The performance of human dental pulp stem cells on different three-dimensional scaffold materials. Biomaterials 2006;27:5658-68.

[19] Bimstein E, Shoshan S. Enhanced healing of tooth-pulp wounds in the dog by enriched collagen solution as a capping agent. Arch Oral Biol 1981;26:97-101. 
[20] Linde A. Dentin matrix proteins: Composition and possible functions in calcification. Anat Rec 1989;224:154-66.

[21] Schour I, Massler M, eds. The teeth. The rat in laboratory investigation, ed. E. Farris and J. Griffith, Lippincott Co.: Philadelphia, 1949.

[22] William L, Charles F, Dorthy F. Odontogenesis of the rat molar. J Dent Res 1953;32:749-71.

[23] Petters RM, Sommer JR. Transgenic animals as models for human disease. Transgenic Res 2000;9:347-51; discussion 345-6.

[24] Ren Y, Maltha JC, Kuijpers-Jagtman AM. The rat as a model for orthodontic tooth movement-a critical review and a proposed solution. Eur J Orthod 2004;26:483-90. 